\title{
Extrasolar planets in stellar multiple systems (Research Note)
}

\author{
T. Roell ${ }^{1}$, R. Neuhäuser ${ }^{1}$, A. Seifahrt ${ }^{1,2,3}$, and M. Mugrauer ${ }^{1}$ \\ 1 Astrophysical Institute and University Observatory Jena, Schillergäßchen 2, 07745 Jena, Germany \\ e-mail: troell@astro.uni-jena.de \\ 2 Physics Department, University of California, Davis, CA 95616, USA \\ 3 Department of Astronomy and Astrophysics, University of Chicago, IL 60637, USA
}

Received 8 September 2011 / Accepted 3 April 2012

\section{ABSTRACT}

\begin{abstract}
Aims. Analyzing exoplanets detected by radial velocity (RV) or transit observations, we determine the multiplicity of exoplanet host stars in order to study the influence of a stellar companion on the properties of planet candidates.

Methods. Matching the host stars of exoplanet candidates detected by radial velocity or transit observations with online multiplicity catalogs in addition to a literature search, 57 exoplanet host stars are identified having a stellar companion.

Results. The resulting multiplicity rate of at least $12 \%$ for exoplanet host stars is about four times smaller than the multiplicity of solar like stars in general. The mass and the number of planets in stellar multiple systems depend on the separation between their host star and its nearest stellar companion, e.g. the planetary mass decreases with an increasing stellar separation. We present an updated overview of exoplanet candidates in stellar multiple systems, including 15 new systems (compared to the latest summary from 2009).
\end{abstract}

Key words. planets and satellites: general - binaries: general - planetary systems

\section{Introduction}

More than 700 extrasolar planet (exoplanet) candidates were discovered so far (Schneider et al. 2011, http://www. exoplanet.eu), but the knowledge of their properties is strongly affected by observational bias and selection effects. Taking the solar system as an archetype, the target lists of exoplanet search programs so far originally consist of mostly single and solar like stars (regarding the spectral type and age). But the first planet candidate detected by the RV technique was found around the primary of the close spectroscopic binary $\gamma$ Cep (Campbell et al. 1988; Hatzes et al. 2003; Neuhäuser et al. 2007), which demonstrates the existence of planets in binaries.

In the last years, imaging campaigns found stellar companions around several dozen exoplanet host stars formerly believed to be single stars (see e.g. Raghavan et al. 2006; Mugrauer \& Neuhäuser 2009, and references therein). Most of these exoplanet candidates are in the S-type orbit configuration (exoplanet surrounding one stellar component of a binary), while the orbit of a planet around both stellar binary components is called P-type orbit. Such circumbinary planets are detectable by measuring eclipse timing variations as done for NN Ser (Beuermann et al. 2010), HW Vir (Lee et al. 2009), DPLeo (Qian et al. 2010), HU Aqr (Qian et al. 2011; Hinse et al. 2012), and UZ For (Dai et al. 2010; Potter et al. 2011). Kepler-16(AB)b, Kepler-34 (AB)b, and Kepler-35 (AB)b are detected by measuring the transit lightcurve and eclipse timing variations (Doyle et al. 2011; Welsh et al. 2012), thus these are confirmed circumbinary planets. Due to a different formation and evolution scenario for planets in a P-type orbit (compared to the more common S-type orbit), this paper only considers exoplanets found in a S-type orbit.

Multiplicity studies, as done by Mugrauer et al. (2007b) or Eggenberger et al. (2007), are looking for stellar companions around exoplanet host stars by direct imaging. As summarized in Mugrauer \& Neuhäuser (2009), these studies found 44 stellar companions around stars previously not known to be multiple, which results in a multiplicity rate of about $17 \%$, while Raghavan et al. (2006) found a host star multiplicity of about $23 \%$. The multiplicity rate of solar like stars ${ }^{1}$ was determined by Raghavan et al. (2010) to $(46 \pm 2) \%$. Duquennoy \& Mayor (1991) measured the multiplicity of 164 nearby G-dwarfs (within 22 pc) to $44 \%$ (57\% considering incompleteness).

\section{Extrasolar planets in stellar multiple systems}

The deuterium burning minimum mass (DBMM) of $13 M_{\mathrm{Jup}}$ is currently the most common criterion to distinguish a brown dwarf from a planet. However, we make use of the Extrasolar Planets Encyclopaedia (EPE) in this paper and thus apply the definition of Schneider et al. (2011) who includes all confirmed substellar companions with a mass of less than $25 M_{\text {Jup }}$ within a $1 \sigma$ uncertainty. Due to a missing publication of the planet detection, the exoplanet candidates GJ $433 \mathrm{~b}, \rho \mathrm{CrBb}$, $91 \mathrm{Aqr} b, v$ Oph b\&c, $\tau$ Gemb, HD 59686 b, HD 106515A b, HD $20781 \mathrm{~b} \& \mathrm{c}$, and HD $196067 \mathrm{~b}$ are not included in this paper. Also, the stellar binary HD 176051, where Muterspaugh et al. (2010) detected the astrometric signal of an exoplanet around one of the two stellar components, is not included in this study: Because the planet was found by ground based astrometric observation (using an optical interferometer), this detection still need to be confirmed by other techniques and the final planetary mass depends on which of the stellar components is the host star.

The multiplicity of an exoplanet host star is defined (in this paper) by either a published common proper motion or an entry in the Catalogue of Components of Double and Multiple Stars

\footnotetext{
1 Defined as all main-sequence stars with a spectral type from F6 to K3 within 25 parsec, see Raghavan et al. (2010).
} 
Table 1. Multiplicity of solar like and exoplanet host stars.

\begin{tabular}{|c|c|c|c|c|}
\hline "Multiple & Single & Double & Triple or higher & Reference \\
\hline \multicolumn{5}{|c|}{$\underline{\text { solar like stars }}$} \\
\hline $46 \%$ & $54 \%$ & $34 \%$ & $9 \%$ & 1 \\
\hline $44 \%$ & $56 \%$ & $38 \%$ & $4 \%$ & 2 \\
\hline \multicolumn{5}{|c|}{ exoplanet host stars } \\
\hline $22.9 \%$ & $77.1 \%$ & $19.8 \%$ & $3.1 \%$ & 3 \\
\hline $17.2 \%$ & $82.8 \%$ & $14.8 \%$ & $2.4 \%$ & 4 \\
\hline $11.95 \%$ & $88.05 \%$ & $9.85 \%$ & $2.1 \%$ & 5 \\
\hline
\end{tabular}

References. 1) Raghavan et al. (2010); 2) Duquennoy \& Mayor (1991); 3) Raghavan et al. (2006); 4) Mugrauer \& Neuhäuser (2009); 5) this work.

(CCDM) by Dommanget \& Nys (2000). In case, the stellar multiplicity is only mentioned in the CCDM, all stellar components were checked on common proper motion using other catalogs (see Appendix A). By searching the literature and matching the host stars of exoplanet candidates detected with transit or RV observations listed in the EPE (date: 2012/02/08) with the CCDM, 57 stellar multiple systems (47 double and 10 triple systems) with at least one exoplanet out of 477 systems in total are identified. The resulting multiplicity rate of about $12 \%$ is less than previously published values (see Table 1). An explanation for that can be the increasing number of transiting exoplanets in the last years, which are included in this paper but excluded by previous studies. The host star multiplicity of transiting exoplanets is most likely still underestimated, because multiplicity studies around such host stars, like done by Daemgen et al. (2009), have just recently started.

The complete list of the 57 multiple systems harboring exoplanet candidates can be found in Tables A.4-A.6. Furthermore, the proper motions of all these stars gathered from online catalogs are shown in Tables A.1-A.3. The latest published summary, done by Mugrauer \& Neuhäuser (2009), listed 44 planetary systems in a stellar multiple system. However, two of these systems are excluded in this study, namely HD 156846 AB (after Reffert \& Quirrenbach 2011, published astrometric mass limits of $m_{\mathrm{pl}}=(10.5 \ldots 660.9) M_{\mathrm{Jup}}$, the EPE planetary status changed to unconfirmed), and $91 \mathrm{Aqr}$ (the planet detection itself is still not published in a refereed paper). In addition to that 42 systems, 15 new systems are listed and marked by the symbol $a$ in Tables A.4-A.6.

\section{Comparison of extrasolar planets in stellar multiple systems and around single stars}

Marcy et al. (2005a) fitted the histogram of all known RV exoplanet minimum masses by a simple power-law and found an exponent of -1.05 for the mass distribution. That exponent is in good agreement with a sample of synthetic exoplanets detectable by current RV observations modeled by Mordasini et al. (2009). In our work, planets currently found by RV or transit observations are analyzed (see Fig. 1). Using also a simple power-law (see Fig. 2), an exponent of -1.03 was found for the mass distribution of all exoplanet candidates, which is similar to the results of previous works. For exoplanet candidates in stellar multiple systems and around single stars, the exponent is -0.97 and -1.04 , respectively. The mean of the planetary masses is about $2.5 M_{\text {Jup }}$ for planets around single stars and 3.1 $M_{\text {Jup }}$ for the case of stellar multiplicity. In addition to the power-law, we also fit a log-normal distribution to the planetary masses. The probability distribution function (PDF) and the

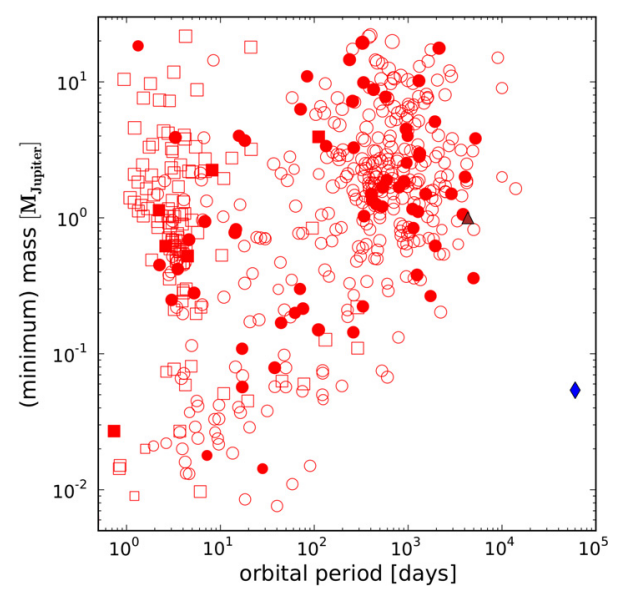

Fig. 1. The (minimum) mass of extrasolar planets detected by RV (circles) or transit (squares) observations over their orbital period. Exoplanets around single stars are shown as open markers, while exoplanets in stellar multiple systems are coded by filled symbols. Jupiter is shown as a filled triangle and the filled diamond marks Neptune.
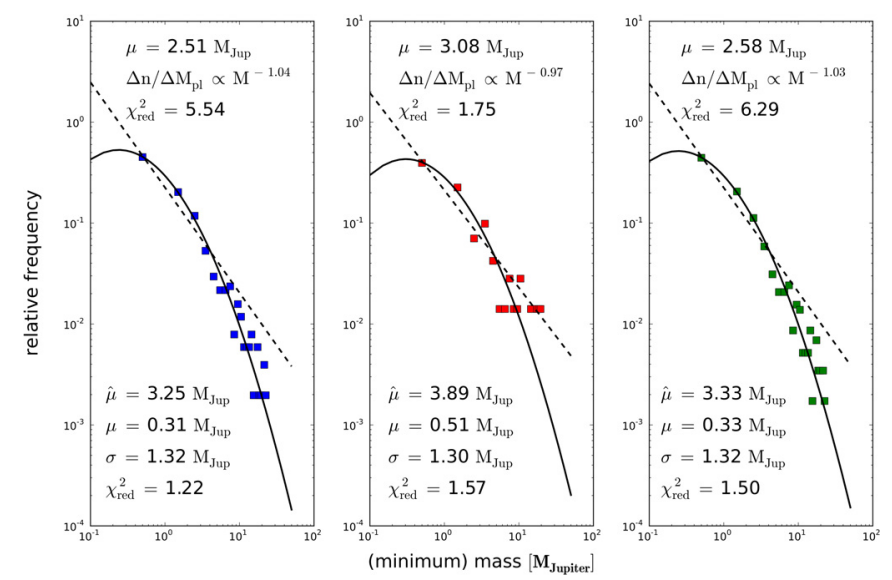

Fig. 2. Mass distribution of exoplanets detected by RV or transit observations around single stars (left column), in stellar multiple systems (middle column), and for all kind of host stars (right column) fitted by a power law (dashed line, upper values) and a log-normal distribution (solid line, lower values).

expectation value $\hat{\mu}$ of a $\log$-normal distribution for a measure $x$ can be calculated by

$\operatorname{PDF}(x, \mu, \sigma)=\mathrm{e}^{-\frac{(\ln x-\mu)^{2}}{2 \sigma^{2}}} /(x \sigma \sqrt{2 \pi}), \quad \hat{\mu}=\mathrm{e}^{\mu+\sigma^{2} / 2}$

where $\mu$ and $\sigma$ are the mean value and the standard deviation of the distribution. To determine the $\chi^{2}$ value (shown in Fig. 2) the Python package "SciPy" (Jones et al. 2001) was used.

As one can see in Fig. 2, the log-normal fit results in a better $\chi_{\text {red }}^{2}$ than the power-law fit. The expectation values for the mass of exoplanets around single stars and in stellar multiple systems differ, hence the power-law as well as the log-normal fit lead to the conclusion that the mass distribution of exoplanets in stellar multiple systems are pushed towards higher planetary masses, compared to the mass distribution of exoplanets around single stars.

However, the statistic of the exoplanet host star multiplicity is still affected by observational bias and selection effects of the originally planet search programs. Most multiplicity studies so far were carried out after the planet detection. Hence, most of the host stars are solar like (regarding the age and spectral type), but they are also originally selected as single stars. To avoid the 
Table 2. Critical semi-major axis $a_{\text {crit }}$ for planets in close stellar binaries, calculated according to Holman \& Wiegert (1999).

\begin{tabular}{lcccccccccc}
\hline \hline Host star & $\begin{array}{c}M_{\text {host }} \\
{\left[M_{\odot}\right]}\end{array}$ & $\begin{array}{c}M_{\text {comp }} \\
{\left[M_{\odot}\right]}\end{array}$ & $\mu_{\text {bin }}$ & $e_{\text {bin }}$ & $\begin{array}{c}a_{\text {bin }} \\
{[\mathrm{AU}]}\end{array}$ & $\begin{array}{c}a_{\text {crit }} \\
{[\mathrm{AU}]}\end{array}$ & $e_{\mathrm{pl}}$ & $\begin{array}{c}a_{\mathrm{pl}} \\
{[\mathrm{AU}]}\end{array}$ & $\begin{array}{c}r_{\mathrm{pl}}^{\text {apastron }} \\
{[\mathrm{AU}]}\end{array}$ & References \\
\hline$\gamma$ Cep A & 1.40 & 0.41 & 0.23 & 0.41 & 20.2 & 3.86 & 0.05 & 2.05 & 2.15 & Neuhäuser et al. (2007) \\
HD 1004 A & 0.70 & 0.42 & 0.38 & 0.40 & 20.0 & 3.38 & 0.39 & 1.60 & 2.28 & Chauvin et al. (2011) \\
HD 196885 A & 1.33 & 0.45 & 0.25 & 0.42 & 21.0 & 3.84 & 0.48 & 2.60 & 3.85 & Chauvin et al. (2011) \\
HD 126614 A & 1.15 & 0.32 & 0.22 & $\leq 0.6$ & 36.2 & $\geq 4.24$ & 0.41 & 2.35 & 3.13 & Howard et al. (2010) \\
HD 19994 A & 1.34 & $0.90^{a}$ & 0.40 & $0.0^{b}$ & $\sim 100^{b}$ & $\sim 31$ & 0.30 & 1.42 & 1.85 & Roell et al. (2011); Mayor et al. (2004) \\
\hline
\end{tabular}

Notes. ${ }^{(a)}$ HD $19994 \mathrm{~B}$ itself is a close stellar binary with a total mass of $M_{\mathrm{BC}}=0.9 M_{\odot}$ (Roell et al. 2011). ${ }^{(b)}$ Values for eccentricity and semi-major axis of HD 19994 are taken from Eggenberger et al. (2004).

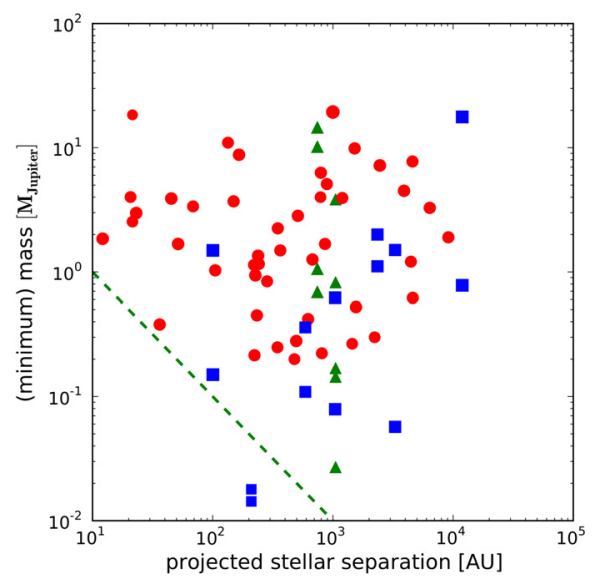

Fig. 3. Planetary (minimum) mass over the projected separation of the exoplanet host star and its nearest stellar companion. The markers represent the number of planets per system (dots ... one planet, squares ... two planets, triangles ... three or more planets). The size of the symbols represent the mass of the exoplanet host star.

adaption of such selection effects, systematic searches for planets in stellar multiple systems, like described in Desidera et al. (2007) or Roell et al. (2010), are needed.

\section{Influence of a close stellar companion on planet properties}

In the previous section, the difference in the mass of exoplanets around single stars and in stellar multiple systems was discussed. In order to unveil the cause of this difference, a closer look on the influence of a stellar companion around the exoplanet host star is advisable. In Fig. 3 we plot the planetary (minimum) mass over the projected separation of the exoplanet host star and its nearest stellar companion. Because all systems analyzed in this paper are hierarchical, the exoplanet host star and its nearest stellar companion can be treated as a binary system. The order of the stellar multiplicity is not relevant, but the planetary minimum mass decreases with an increasing projected stellar separation (dashed line in Fig. 3). Furthermore, multi-planet systems are only present in stellar systems with a projected stellar separation larger than about $100 \mathrm{AU}$ and up to now, no planet was found in a stellar binary with a projected separation of less than $10 \mathrm{AU}$. The two planets below the dashed line in Fig. 3 are the planetary system around GJ $667 \mathrm{C}$, a component of a hierarchical triple star system at a distance of $7 \mathrm{pc}$. The true semi-major axis is likely larger than the measured projected separation and the true planetary mass could also be larger than the measured minimum mass. These observational bias effects could explain, why GJ $667 \mathrm{C}$ is the only system left of that dashed line in Fig. 3.

Holman \& Wiegert (1999) determine a formula to calculate the critical semi-major axis $a_{\text {crit }}$ for a stable planetary orbit

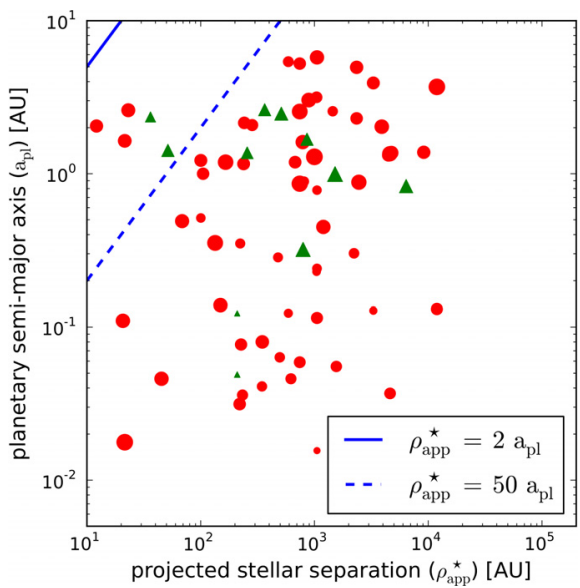

Fig. 4. Planetary semi-major axis over the projected stellar separation (dots ... stellar binary, triangles ... triple star). The five systems in the upper left $\left(\rho_{\text {app }}^{\star}<50 a_{\mathrm{pl}}\right)$ are shown in more detail in Table 2. The size of the symbols represent the (minimum) mass of the exoplanet.

coplanar to the stellar orbit with the semi-major axis $a_{\text {bin }}$, which varies from $a_{\text {crit }} \simeq(0.02 \ldots 0.45) a_{\text {bin }}$, depending on the mass ratio $\mu_{\text {bin }}$ and the eccentricity $e_{\text {bin }}$ of the stellar binary. Table 2 listed the five systems, where the apparent separation is less than 50 times the planetary semi-major axis (see Fig. 4) including the corresponding critical semi-major axis. Except for the exoplanet HD $196885 \mathrm{Ab}$, which grazes an "unstable region" during the apastron passage, all these systems are clearly stable. However, considering the age of the F8V star HD 196885 A of $2.0 \pm 0.5 \mathrm{Gyr}$ (Correia et al. 2008), the planetary system can also be regarded as long-term stable.

\section{Summary}

Analyzing the host star multiplicty of exoplanets detected by RV or transit observations, 57 exoplanet host stars with stellar companions are identified and presented in the Appendix A, including 15 new systems (compared to the latest published summary in 2009). The resulting multiplicity rate for exoplanet host stars of at least $12 \%$ is about four times smaller than the multiplicity of solar like stars. No planet is found so far in stellar binaries with a projected separation of less than $10 \mathrm{AU}$ and multi-planet systems were only found in stellar systems with a projected separation larger than $100 \mathrm{AU}$. The planetary (minimum) mass decreases with an increasing projected stelllar separation.

Acknowledgements. T.R. and R.N. thank the DFG (Deutsche Forschungsgemeinschaft) for financial support under the project numbers NE515/23-1, NE 515/30-1, and NE 515/33-1 (SPP 1385: "First ten million years of the solar systems"). A.S. acknowledge support from the National Science Foundation under grant NSFAST-0708074. This research has made use of the SIMBAD database and the VizieR catalog access tool, both operated at CDS, Strasbourg, France. 


\section{References}

Anglada-Escudé, G., Arriagada, P., Vogt, S. S., et al. 2012, ApJ, 751, L16 Bakos, G. Á., Pál, A., Latham, D. W., Noyes, R. W., \& Stefanik, R. P. 2006, ApJ, 641, L57

Bakos, G. Á., Noyes, R. W., Kovács, G., et al. 2007, ApJ, 656, 552 Beuermann, K., Hessman, F. V., Dreizler, S., et al. 2010, A\&A, 521, L60 Bouchy, F., Udry, S., Mayor, M., et al. 2005, A\&A, 444, L15

Burke, C. J., McCullough, P. R., Valenti, J. A., et al. 2007, ApJ, 671, 2115 Butler, R. P., Marcy, G. W., Williams, E., Hauser, H., \& Shirts, P. 1997, ApJ, 474, L115

Butler, R. P., Marcy, G. W., Fischer, D. A., et al. 1999, ApJ, 526, 916 Butler, R. P., Tinney, C. G., Marcy, G. W., et al. 2001, ApJ, 555, 410 Butler, R. P., Marcy, G. W., Vogt, S. S., et al. 2003, ApJ, 582, 455 Butler, R. P., Wright, J. T., Marcy, G. W., et al. 2006, ApJ, 646, 505 Campbell, B., Walker, G. A. H., \& Yang, S. 1988, ApJ, 331, 902 Chauvin, G., Lagrange, A.-M., Udry, S., et al. 2006, A\&A, 456, 1165 Chauvin, G., Lagrange, A., Udry, S., \& Mayor, M. 2007, A\&A, 475, 723

Chauvin, G., Beust, H., Lagrange, A.-M., \& Eggenberger, A. 2011, A\&A, 528, A8

Cochran, W. D., Hatzes, A. P., Butler, R. P., \& Marcy, G. W. 1997, ApJ, 483, 457

Correia, A. C. M., Udry, S., Mayor, M., et al. 2008, A\&A, 479, 271

Daemgen, S., Hormuth, F., Brandner, W., et al. 2009, A\&A, 498, 567

Dai, Z.-B., Qian, S.-B., Fernández Lajús, E., \& Baume, G. L. 2010, MNRAS, 409, 1195

Desidera, S., \& Barbieri, M. 2007, A\&A, 462, 345

Desidera, S., Gratton, R., Endl, M., et al. 2007 [arXiv:0705. 3141]

Desidera, S., Carolo, E., Gratton, R., et al. 2011, A\&A, 533, A90

Dommanget, J., \& Nys, O. 2000, A\&A, 363, 991

Doyle, L. R., Carter, J. A., Fabrycky, D. C., et al. 2011, Science, 333, 1602

Dumusque, X., Lovis, C., Ségransan, D., et al. 2011, A\&A, 535, A55

Duquennoy, A., \& Mayor, M. 1991, A\&A, 248, 485

Eggenberger, A., Udry, S., \& Mayor, M. 2003, in Scientific Frontiers in Research on Extrasolar Planets, ed. D. Deming, \& S. Seager, ASP Conf. Ser., 294, 43

Eggenberger, A., Udry, S., \& Mayor, M. 2004, A\&A, 417, 353

Eggenberger, A., Mayor, M., Naef, D., et al. 2006, A\&A, 447, 1159

Eggenberger, A., Udry, S., Chauvin, G., et al. 2007, A\&A, 474, 273

Fischer, D., Driscoll, P., Isaacson, H., et al. 2009, ApJ, 703, 1545

Fischer, D. A., Marcy, G. W., Butler, R. P., Vogt, S. S., \& Apps, K. 1999, PASP, 111,50

Fischer, D. A., Marcy, G. W., Butler, R. P., et al. 2001, ApJ, 551, 1107

Fischer, D. A., Butler, R. P., Marcy, G. W., Vogt, S. S., \& Henry, G. W. 2003a, ApJ, 590, 1081

Fischer, D. A., Marcy, G. W., Butler, R. P., et al. 2003b, ApJ, 586, 1394

Fischer, D. A., Laughlin, G., Marcy, G. W., et al. 2006, ApJ, 637, 1094

Fischer, D. A., Vogt, S. S., Marcy, G. W., et al. 2007, ApJ, 669, 1336

Forveille, T., Bonfils, X., Lo Curto, G., et al. 2011, A\&A, 526, A141

Ginski, C., Mugrauer, M., Seeliger, M., \& Eisenbeiss, T. 2012, MNRAS, 421, 2498

Guenther, E. W., Hartmann, M., Esposito, M., et al. 2009, A\&A, 507, 1659

Han, I., Lee, B. C., Kim, K. M., et al. 2010, A\&A, 509, A24

Hatzes, A. P., Cochran, W. D., Endl, M., et al. 2003, ApJ, 599, 1383

Hinse, T. C., Lee, J. W., Goździewski, K., et al. 2012, MNRAS, 420, 3609

Høg, E., Fabricius, C., Makarov, V. V., et al. 2000, A\&A, 355, L27

Holman, M. J., \& Wiegert, P. A. 1999, AJ, 117, 621

Howard, A. W., Johnson, J. A., Marcy, G. W., et al. 2010, ApJ, 721, 1467

Johnson, J. A., Marcy, G. W., Fischer, D. A., et al. 2006, ApJ, 652, 1724

Jones, E., Oliphant, T., Peterson, P., et al. 2001, SciPy: Open source scientific tools for Python

Jones, H. R. A., Paul Butler, R., Marcy, G. W., et al. 2002, MNRAS, 337, 1170 Jones, H. R. A., Butler, R. P., Tinney, C. G., et al. 2006, MNRAS, 369, 249

Kharchenko, N. V. 2001, Kinematika i Fizika Nebesnykh Tel, 17, 409

Korzennik, S. G., Brown, T. M., Fischer, D. A., Nisenson, P., \& Noyes, R. W. 2000, ApJ, 533, L147

Latham, D. W., Stefanik, R. P., Mazeh, T., Mayor, M., \& Burki, G. 1989, Nature, 339, 38

Lee, J. W., Kim, S., Kim, C., et al. 2009, AJ, 137, 3181

Liu, Y.-J., Sato, B., Zhao, G., et al. 2008, ApJ, 672, 553
Lo Curto, G., Mayor, M., Clausen, J. V., et al. 2006, A\&A, 451, 345

Lo Curto, G., Mayor, M., Benz, W., et al. 2010, A\&A, 512, A48

Lovis, C., Mayor, M., Bouchy, F., et al. 2005, A\&A, 437, 1121

Lowrance, P. J., Kirkpatrick, J. D., \& Beichman, C. A. 2002, ApJ, 572, L79

Marcy, G. W., Butler, R. P., \& Vogt, S. S. 2000, ApJ, 536, L43

Marcy, G. W., Butler, R. P., Fischer, D. A., et al. 2002, ApJ, 581, 1375

Marcy, G., Butler, R. P., Fischer, D., et al. 2005a, Prog. Theor. Phys. Suppl., 158, 24

Marcy, G. W., Butler, R. P., Vogt, S. S., et al. 2005b, ApJ, 619, 570

Mayor, M., Udry, S., Naef, D., et al. 2004, A\&A, 415, 391

McAlister, H. A., Hartkopf, W. I., Hutter, D. J., \& Franz, O. G. 1987, AJ, 93, 688

McArthur, B. E., Endl, M., Cochran, W. D., et al. 2004, ApJ, 614, L81

Mordasini, C., Alibert, Y., Benz, W., \& Naef, D. 2009, A\&A, 501, 1161

Moutou, C., Hébrard, G., Bouchy, F., et al. 2009, A\&A, 498, L5

Mugrauer, M., \& Neuhäuser, R. 2005, MNRAS, 361, L15

Mugrauer, M., \& Neuhäuser, R. 2009, A\&A, 494, 373

Mugrauer, M., Neuhäuser, R., Mazeh, T., Alves, J., \& Guenther, E. 2004a, A\&A, 425, 249

Mugrauer, M., Neuhäuser, R., Mazeh, T., Guenther, E., \& Fernández, M. 2004b, AN, 325, 718

Mugrauer, M., Neuhäuser, R., Seifahrt, A., Mazeh, T., \& Guenther, E. 2005, A\&A, 440, 1051

Mugrauer, M., Neuhäuser, R., Mazeh, T., et al. 2006a, AN, 327, 321

Mugrauer, M., Seifahrt, A., Neuhäuser, R., \& Mazeh, T. 2006b, MNRAS, 373, L31

Mugrauer, M., Neuhäuser, R., \& Mazeh, T. 2007a, A\&A, 469, 755

Mugrauer, M., Seifahrt, A., \& Neuhäuser, R. 2007b, MNRAS, 378, 1328

Muterspaugh, M. W., Lane, B. F., Kulkarni, S. R., et al. 2010, AJ, 140, 1657

Naef, D., Latham, D. W., Mayor, M., et al. 2001a, A\&A, 375, L27

Naef, D., Mayor, M., Pepe, F., et al. 2001b, A\&A, 375, 205

Naef, D., Mayor, M., Korzennik, S. G., et al. 2003, A\&A, 410, 1051

Naef, D., Mayor, M., Lo Curto, G., et al. 2010, A\&A, 523, A15

Neuhäuser, R., Mugrauer, M., Fukagawa, M., Torres, G., \& Schmidt, T. 2007, A\&A, 462, 777

Patience, J., White, R. J., Ghez, A. M., et al. 2002, ApJ, 581, 654

Pont, F., Hébrard, G., Irwin, J. M., et al. 2009, A\&A, 502, 695

Porto de Mello, G. F., \& da Silva, L. 1997, ApJ, 476, L89

Potter, S. B., Romero-Colmenero, E., Ramsay, G., et al. 2011, MNRAS, 416, 2202

Qian, S., Liao, W., Zhu, L., et al. 2010, MNRAS, 401, L34

Qian, S.-B., Liu, L., Liao, W.-P., et al. 2011, MNRAS, 414, L16

Queloz, D., Mayor, M., Weber, L., et al. 2000, A\&A, 354, 99

Queloz, D., Anderson, D., Collier Cameron, A., et al. 2010, A\&A, 517, L1

Raghavan, D., Henry, T. J., Mason, B. D., et al. 2006, ApJ, 646, 523

Raghavan, D., McAlister, H. A., Henry, T. J., et al. 2010, ApJS, 190, 1

Reffert, S., \& Quirrenbach, A. 2011, A\&A, 527, A140

Roell, T., Neuhäuser, R., \& Seifahrt, A. 2010, in EAS Publ. Ser. 42, ed. K. Gożdziewski, A. Niedzielski, \& J. Schneider, 179

Roell, T., Seifahrt, A., Neuhäuser, R., Köhler, R., \& Bean, J. 2011, in EAS Publ. Ser. 45, ed. C. Turon, F. Meynadier, \& F. Arenou, 429

Roeser, S., Demleitner, M., \& Schilbach, E. 2010, AJ, 139, 2440

Santos, N. C., Mayor, M., Naef, D., et al. 2001, A\&A, 379, 999

Schneider, J., Dedieu, C., Le Sidaner, P., Savalle, R., \& Zolotukhin, I. 2011, A\&A, 532, A79

Tinney, C. G., Butler, R. P., Marcy, G. W., et al. 2002, ApJ, 571, 528

Udry, S., Mayor, M., Naef, D., et al. 2000, A\&A, 356, 590

van Leeuwen, F. 2008, VizieR Online Data Catalog, 1311

Vogt, S. S., Marcy, G. W., Butler, R. P., \& Apps, K. 2000, ApJ, 536, 902

Vogt, S. S., Butler, R. P., Marcy, G. W., et al. 2005, ApJ, 632, 638

Welsh, W. F., Orosz, J. A., Carter, J. A., et al. 2012, Nature

Zacharias, N., Monet, D. G., Levine, S. E., et al. 2004, BAAS, 36, 1418

Zacharias, N., Finch, C., Girard, T., et al. 2010, AJ, 139, 2184

Zucker, S., Naef, D., Latham, D. W., et al. 2002, ApJ, 568, 363

Zucker, S., Mazeh, T., Santos, N. C., Udry, S., \& Mayor, M. 2003, A\&A, 404, 775

Zucker, S., Mazeh, T., Santos, N. C., Udry, S., \& Mayor, M. 2004, A\&A, 426, 695 
T. Roell et al.: Extrasolar planets in stellar multiple systems $(R N)$

\section{Appendix A: Updated tables of extrasolar planets in stellar multiple systems}

Table A.1. Exoplanet host stars having a common proper motion with another star. The ID of the host stars is the same as it is used in the EPE and the planet status is coded by R ... published in a Refereed paper, S ... Submitted to a professional journal, and C ... announced by astronomers in professional Conferences. A planet status followed by the letter R in brackets means, the status on the EPE is not up-to-date and the planet detection is already published in a refereed paper. The fourth and fifth column show the proper motion listed in the catalog mentioned in the last column. For easy identification of the stellar companions in the online catalogs, the third column contains either the separation from the primary as calculated by the used online catalog or the latest separation measurement in case of published relative astrometric measurements.

\begin{tabular}{|c|c|}
\hline CCDM & Catalog of Components of Double and Multiple stars, Dommanget \& Nys (2000). \\
\hline ASCC $-2.5 \mathrm{~V} 3 \ldots$ & All-sky Compiled Catalogue of 2.5 million stars (3rd version, 2009), Kharchenko (2001). \\
\hline HIP-2 . & Hipparcos, the New Reduction, van Leeuwen (2008). \\
\hline Nomad-1 ... & Naval Observatory Merged Astrometric Dataset, Zacharias et al. (2004). \\
\hline UCAC-3. & Third U.S. Naval Observatory CCD Astrograph Catalog, Zacharias et al. (2010). \\
\hline PPMXL . & $\begin{array}{l}\text { The PPMXL catalog of positions and proper motions on the ICRS. Combining USNO-B1.0 } \\
\text { and the two Micron All Sky Survey (2MASS)., Roeser et al. (2010). }\end{array}$ \\
\hline Tycho-2 & The Tycho-2 Catalogue of the 2.5 Million Brightest Stars, Høg et al. (2000). \\
\hline
\end{tabular}

\begin{tabular}{lccccc}
\hline \hline System & EPE planet status & $r\left[^{\prime \prime}\right]$ & $\mu_{\alpha} \cos \delta[\mathrm{mas} /$ year $]$ & $\mu_{\delta}[\mathrm{mas} /$ year $]$ & Catalogue \\
\hline 11 Com A & $\mathrm{R}$ & 9 & $-109.37 \pm 1.26$ & $89.25 \pm 0.75$ & ASCC-2.5-V3 \\
11 Com B & - & - & $-109 \pm$ n.s. & $85 \pm$ n.s. & CCDM \\
& - & - & $-133.39 \pm 0.82$ & $-163.08 \pm 0.85$ & ASCC-2.5-V3 \\
16 Cyg A & $\mathrm{R}$ & 39.44 & $-147.77 \pm 0.85$ & $-158.39 \pm 0.76$ & ASCC-2.5-V3
\end{tabular}

$16 \mathrm{Cyg} \mathrm{C} \quad-\quad$ close C component about 3.4" away from A, see Patience et al. (2002)

30 Ari A

30 Ari B

$55 \mathrm{Cnc} \mathrm{A}$

$55 \mathrm{CncB}$

91 Aqr A

91 Aqr B

91 Aqr C

91 Aqr D

91 Aqr E

$\gamma$ Cep A

$\gamma$ Cep B

$\gamma^{1}$ Leo A

$\gamma^{1}$ Leo B

$\gamma^{1}$ Leo C

$\gamma^{1}$ Leo D

$\tau$ Boo A

$\tau$ Boo B

$v$ And $\mathrm{A}$

$v$ And B

$v$ And C

$v$ And D

GJ $667 \mathrm{AB}$

GJ 667 C
$-$

$\overline{\mathrm{R}}$

$\mathrm{R}$

$-$

S

$-$

$-$

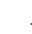

$137.66 \pm 0.74$

$-14.98 \pm 0.91$

A itself is a spectroscopic binary, see Guenther et al. (2009)

$36.86 \quad 145.33 \pm 1.21 \quad-12.86 \pm 0.94$

$-234.4 \pm 0.7$

84.16

$-485.4 \pm 0.9$

$-234.0 \pm 5.0$

$370.70 \pm 0.63$

51.93

52.03

106.67

88.05

$373.00 \pm 2.41$

$370.00 \pm 1.87$

$-16.22 \pm 0.7$

$-18.46 \pm 2.41$

$18.3 \pm 8.4$

$-20.34 \pm 0.98$
$-10.5 \pm 7.8$

$-9.4 \pm 8.7$

$-25.2 \pm 7.8$

$-48.8 \pm 0.4$

$127.1 \pm 0.4$

0.02 imaged directly by Neuhäuser et al. (2007)

-
4.33
325.1

325.15

$306.35 \pm 3.27$

$309.60 \pm 1.32$

$-160.77 \pm 2.30$

$-501.30 \pm 1.05$

$-152.91 \pm 0.75$

$-10.01 \pm 1.53$

$-41.97 \pm 1.28$

$-22.85 \pm 1.21$

$-479.53 \pm 0.16$

$53.49 \pm 0.13$

ASCC-2.5-V3

ASCC-2.5-V3

Nomad-1

Nomad-1

ASCC-2.5 V3

ASCC- $2.5 \mathrm{~V} 3$

ASCC-2.5 V3

Nomad-1

Nomad-1

Nomad-1

ASCC- $2.5 \mathrm{~V} 3$

ASCC- $2.5 \mathrm{~V} 3$

ASCC-2.5 V3

ASCC-2.5 V3

HIP-2

2.8 confirmed by Duquennoy \& Mayor (1991)

$\begin{array}{lcr}- & -172.5 \pm 0.5 & -381.0 \pm 0.4 \\ 55 & \text { confirmed by Lowrance et al. }(2002)\end{array}$

$110.27 \quad-9.6 \pm 2.3 \quad-3.5 \pm 2$.

$\begin{array}{lll}273.20 & 16.3 \pm 0.7 & -4.7 \pm 0.6\end{array}$

$1129.76 \pm 9.72 \quad-77.02 \pm 4.67$

$1171.69 \pm 2.70 \quad-168.80 \pm 2.79$

$1161.4 \pm 2.3$

$-172.3 \pm 2.3$

$1049.0 \pm$ n.s.

$-91.0 \pm$ n.s.

$1155.0 \pm 7.2 \quad-214.4 \pm 9.5$

Nomad-1

Nomad-1

Nomad-1

HIP-2

ASCC-2.5 V3

PPMXL

UCAC-3

NOMAD-1

GJ $667 \mathrm{C}$ has not a common proper motion within the measurement errors, but it can be ruled out as a background object.

The large spread in the proper motion measurements can be possibly explained by the fact, that the $\mathrm{AB}$ component influences the relative astrometric measurements of the $(\mathrm{AB}) \& \mathrm{C}$ pair.

\begin{tabular}{lccccc} 
GJ 676 A & $\mathrm{R}$ & - & $-259.2 \pm 1.4$ & $-185.6 \pm 0.9$ & Nomad-1 \\
GJ 676 B & - & 48.98 & $-254.0 \pm 8.0$ & $-156.0 \pm 23.0$ & Nomad-1 \\
& & 47.78 & $-294.6 \pm 7.4$ & $-186.8 \pm 7.0$ & UCAC-3 \\
GJ 3021 A & $\mathrm{R}$ & - & $433.8 \pm 0.5$ & $-57.9 \pm 0.4$ & Nomad-1 \\
GJ 3021 B & - & 3.86 & confirmed by Chauvin et al. $(2006)$ & \\
Gl 86 A & $\mathrm{R}$ & - & $2183.43 \pm 3.20$ & $661.70 \pm 3.50$ & ASCC-2.5-V3 \\
Gl 86 B & - & 1.93 & confirmed by Mugrauer \& Neuhäuser (2005) & \\
\hline
\end{tabular}


Table A.1. continued.

\begin{tabular}{|c|c|c|c|c|c|}
\hline System & EPE planet status & $\overline{r\left[{ }^{\prime \prime}\right]}$ & $\mu_{\alpha} \cos \delta[$ mas/year $]$ & $\mu_{\delta}[$ mas/year $]$ & Catalogue \\
\hline HAT-P-1 A & - & - & $30.0 \pm 0.6$ & $-42.3 \pm 1.3$ & UCAC-3 \\
\hline HAT-P-1 B & $\mathrm{R}$ & 10.93 & $32.6 \pm 0.8$ & $-43.2 \pm 1.9$ & UCAC-3 \\
\hline WASP-8 A & $S(R)$ & - & $109.62 \pm 2.05$ & $10.02 \pm 1.46$ & ASCC-2.5 V3 \\
\hline WASP-8 B & - & \multicolumn{3}{|c|}{ confirmed by Queloz et al. (2010) } & \\
\hline WASP-8 C & - & 140.93 & $50.33 \pm 1.39$ & $-28.65 \pm 1.08$ & ASCC-2.5 V3 \\
\hline $\mathrm{XO}-2 \mathrm{~A}$ & $C(R)$ & - & $-34.72 \pm 2.50$ & $-153.61 \pm 2.40$ & ASCC-2.5 V3 \\
\hline $\mathrm{XO}-2 \mathrm{~B}$ & - & 30.05 & $-33.02 \pm 2.89$ & $-154.11 \pm 2.70$ & ASCC-2.5 V3 \\
\hline HD $142 \mathrm{~A}$ & $\mathrm{R}$ & - & $575.2 \pm 0.4$ & $-39.9 \pm 0.5$ & Nomad-1 \\
\hline HD 142 B & - & 4.10 & \multicolumn{2}{|c|}{ confirmed by Eggenberger et al. (2007) } & \\
\hline HD $3651 \mathrm{~A}$ & $\mathrm{R}$ & - & $-460.22 \pm 0.89$ & $-370.22 \pm 0.75$ & ASCC-2.5 V3 \\
\hline HD 3651 B & - & 43.07 & \multicolumn{2}{|c|}{ confirmed by Mugrauer et al. (2006b) } & \\
\hline HD $3651 \mathrm{C}$ & - & 168.26 & $10.81 \pm 2.00$ & $-1.48 \pm 2.40$ & ASCC-2.5 V3 \\
\hline HD 7449 A & $\mathrm{R}$ & - & $-161.6 \pm 0.6$ & $-138.9 \pm 0.5$ & PPMXL \\
\hline HD 7449 B & & 60.20 & $-162.5 \pm 6.1$ & $-137.5 \pm 6.1$ & PPMXL \\
\hline HD $16141 \mathrm{~A}$ & $\mathrm{R}$ & - & $-155.33 \pm 1.19$ & $-437.42 \pm 1.23$ & ASCC-2.5 V3 \\
\hline HD 16141 B & - & 6.20 & \multicolumn{2}{|c|}{ confirmed by Mugrauer et al. (2005) } & \\
\hline HD 11964 A & $\mathrm{R}$ & - & $-366.10 \pm 0.3$ & $-240.83 \pm 0.62$ & ASCC-2.5 V3 \\
\hline HD 11964 B & - & 30.68 & $-369.98 \pm 4.34$ & $-245.47 \pm 3.13$ & ASCC-2.5 V3 \\
\hline HD 19994 A & $\mathrm{R}$ & - & $194.56 \pm 0.37$ & $-69.01 \pm 0.30$ & HIP-2 \\
\hline HD 19994 BC & - & $\begin{array}{l}2.30 \\
\text { close } \mathrm{C}\end{array}$ & \multicolumn{3}{|c|}{$\begin{array}{l}\text { confirmed by Duquennoy \& Mayor (1991) } \\
\text { omponent around B found by Roell et al. (2011) }\end{array}$} \\
\hline HD $20782 \mathrm{~A}$ & $\mathrm{R}$ & - & $349.85 \pm 0.80$ & $-65.00 \pm 1.07$ & ASCC-2.5 V3 \\
\hline HD 20782 B & - & 253.71 & $349.76 \pm 1.13$ & $-68.44 \pm 1.57$ & ASCC-2.5 V3 \\
\hline HD $27442 \mathrm{~A}$ & $\mathrm{R}$ & - & $-48.09 \pm 0.80$ & $-166.69 \pm 0.77$ & ASCC-2.5 V3 \\
\hline HD 27442 B & - & 13.06 & \multicolumn{2}{|c|}{ confirmed by Chauvin et al. (2006) } & \\
\hline HD $28254 \mathrm{~A}$ & $S(R)$ & - & \multirow{2}{*}{\multicolumn{2}{|c|}{$\begin{array}{c}-66.9 \pm 0.6 \quad-144.0 \pm 0.6 \\
\text { confirmed by Naef et al. }(2010)\end{array}$}} & Nomad-1 \\
\hline HD 28254 B & - & 4.3 & & & \\
\hline HD 38529 A & $\mathrm{R}$ & - & \multirow{3}{*}{\multicolumn{2}{|c|}{$\begin{array}{cc}-79.22 \pm 1.02 & -142.22 \pm 1.00 \\
-81.79 \pm 14.50 & -117.47 \pm 14.80 \\
\text { nent confirmed by Raghavan et al. }(2006)\end{array}$}} & ASCC-2.5 V3 \\
\hline HD 38529 B & - & 283.72 & & & ASCC-2.5 V3 \\
\hline & & B comp & & & \\
\hline HD $40979 \mathrm{~A}$ & $\mathrm{R}$ & - & \multirow{3}{*}{$\begin{array}{l}96.40 \pm 1.19 \\
94.08 \pm 1.85\end{array}$} & $-153.36 \pm 0.70$ & ASCC-2.5 V3 \\
\hline HD 40979 BC & - & 193.77 & & $-153.11 \pm 1.65$ & ASCC-2.5 V3 \\
\hline & - & C comp & & rom B found by Mugr & auer et al. (2007a) \\
\hline HD $41004 \mathrm{~A}$ & $\mathrm{R}$ & - & \multirow{2}{*}{$\begin{array}{l}-41.72 \pm 1.19 \\
-42.25 \pm 1.08\end{array}$} & $64.87 \pm 1.34$ & ASCC-2.5 V3 \\
\hline HD 41004 B & $\mathrm{R}$ & 1.19 & & $65.16 \pm 1.12$ & ASCC-2.5 V3 \\
\hline HD $46375 \mathrm{~A}$ & $\mathrm{R}$ & - & \multirow{2}{*}{\multicolumn{2}{|c|}{$\begin{array}{cc}114.2 \pm 0.9 & -96.7 \pm 0.7 \\
\text { confirmed by Mugrauer et al. }(2006 a)\end{array}$}} & Nomad-1 \\
\hline HD 46375 B & & 10.35 & & & \\
\hline HD $65216 \mathrm{~A}$ & $\mathrm{R}$ & - & \multirow{3}{*}{\multicolumn{2}{|c|}{$\begin{array}{l}-122.66 \pm 1.34 \quad 145.77 \pm 1.15 \\
\text { confirmed by Mugrauer et al. }(2007 \mathrm{~b}) \\
\text { nent } 0.17^{\prime \prime} \text { away from B found by Mugra }\end{array}$}} & ASCC-2.5 V3 \\
\hline HD 65216 BC & - & 7.14 & & & \\
\hline & & C comp & & & et al. (2007b) \\
\hline HD $75289 \mathrm{~A}$ & $\mathrm{R}$ & - & \multirow{2}{*}{\multicolumn{2}{|c|}{$\begin{array}{cc}-19.90 \pm 0.66 & -228.13 \pm 0.73 \\
\text { confirmed by Mugrauer et al. }(2004 \mathrm{a})\end{array}$}} & ASCC-2.5 V3 \\
\hline HD 75289 B & - & 21.47 & & & \\
\hline HD 80606 A & $\mathrm{R}$ & - & \multirow{2}{*}{$\begin{array}{l}56.84 \pm 1.37 \\
51.95 \pm 1.32\end{array}$} & $10.02 \pm 1.71$ & ASCC-2.5 V3 \\
\hline HD 80606 B & & 20.18 & & $9.97 \pm 1.77$ & ASCC-2.5 V3 \\
\hline HD 89744 A & $\mathrm{R}$ & - & \multirow{2}{*}{\multicolumn{2}{|c|}{$\begin{array}{cc}-120.12 \pm 0.85 & -138.66 \pm 0.76 \\
\text { confirmed by Mugrauer et al. }(2004 \mathrm{~b})\end{array}$}} & \multirow[t]{2}{*}{ ASCC- $2.5 \mathrm{~V} 3$} \\
\hline HD 89744 B & - & 62.99 & & & \\
\hline HD 99491 (A) & - & - & $-725.22 \pm 0.64$ & $180.30 \pm 0.72$ & ASCC-2.5 V3 \\
\hline HD 99492 (B) & $\mathrm{R}$ & 33.27 & $-727.57 \pm 1.55$ & $186.47 \pm 1.55$ & ASCC-2.5 V3 \\
\hline HD 99492 (C) & - & 204.56 & $5.36 \pm 1.98$ & $-13.72 \pm 2.00$ & ASCC-2.5 V3 \\
\hline HD $101930 \mathrm{~A}$ & $\mathrm{R}$ & - & $15.42 \pm 1.30$ & $348.29 \pm 1.27$ & ASCC-2.5 V3 \\
\hline HD $101930 \mathrm{~B}$ & - & 69.89 & $24.98 \pm 4.01$ & $351.54 \pm 2.35$ & ASCC-2.5 V3 \\
\hline & - & B comp & nent confirmed by $\mathrm{Mu}$ & grauer et al. (2007b) & \\
\hline
\end{tabular}


Table A.1. continued.

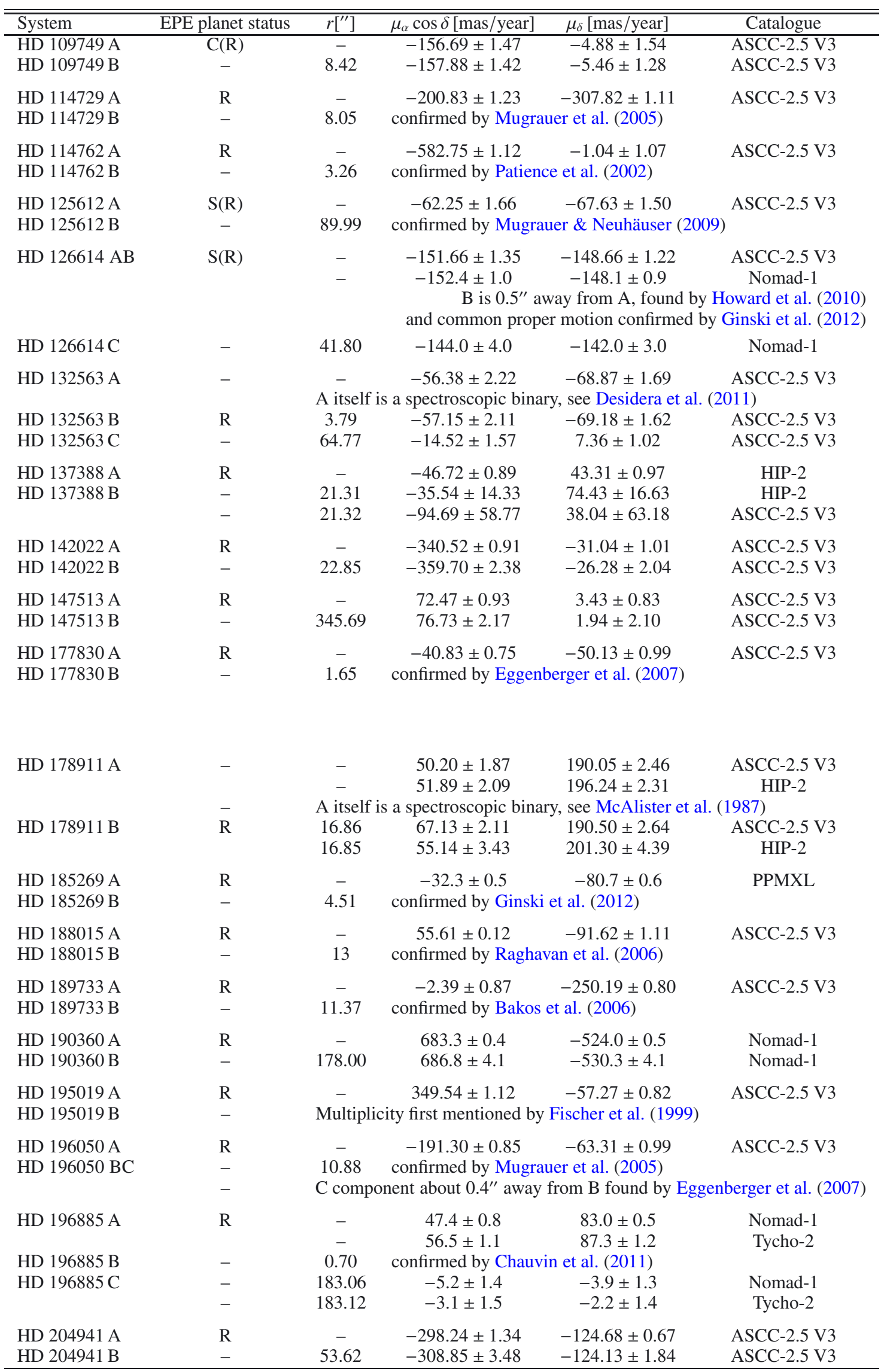


Table A.1. continued.

\begin{tabular}{lccccc}
\hline \hline System & EPE planet status & $r\left[^{\prime \prime}\right]$ & $\mu_{\alpha} \cos \delta[\mathrm{mas} /$ year $]$ & $\mu_{\delta}[\mathrm{mas} /$ year] & Catalogue \\
\hline HD 212301 A & $\mathrm{S}(\mathrm{R})$ & - & $79.12 \pm 1.04$ & $-92.37 \pm 0.99$ & ASCC-2.5 V3 \\
HD 212301 B & - & 4.43 & confirmed by Mugrauer \& Neuhäuser (2009) \\
HD 213240 A & $\mathrm{R}$ & - & $-135.1 \pm 0.6$ & $-194.0 \pm 0.4$ & Nomad-1 \\
HD 213240 B & - & 21.94 & $65.1 \pm 5.0$ & $-10.8 \pm 1.5$ & Nomad-1 \\
HD 213240 C & - & 95.69 & confirmed by Mugrauer et al. (2005) & \\
HD 222582 A & $\mathrm{R}$ & - & $-145.4 \pm 1.2$ & $-111.0 \pm 0.8$ & Nomad-1 \\
HD 222582 B & - & 109.42 & $-147.5 \pm 4.4$ & $-114.2 \pm 4.4$ & Nomad-1 \\
\hline
\end{tabular}

Table A.2. Exoplanet host stars listed in the CCDM, but unlikely a common proper motion pair (same columns as in A.1).

\begin{tabular}{|c|c|c|c|c|c|}
\hline System & EPE planet status & $\overline{c\left[{ }^{\prime \prime}\right]}$ & $\mu_{\alpha} \cos \delta[$ mas/year $]$ & $\mu_{\delta}[\mathrm{mas} /$ year $]$ & Catalogue \\
\hline 6 Lyn A & $\mathrm{R}$ & - & $-30.0 \pm 0.8$ & $-338.8 \pm 0.6$ & Nomad-1 \\
\hline 6 Lyn B & - & 169.91 & $3.6 \pm 1.6$ & $7.2 \pm 1.6$ & Nomad-1 \\
\hline 18 Del A & $\mathrm{R}$ & - & $-47.9 \pm 0.7$ & $-34.3 \pm 0.3$ & Nomad-1 \\
\hline 18 Del B & - & 197.34 & $10.8 \pm 1.1$ & $-12.6 \pm 1.2$ & Nomad-1 \\
\hline 18 Del C & - & 235.69 & $-6.3 \pm 1.6$ & $-6.4 \pm 3.2$ & Nomad-1 \\
\hline 61 Vir A & $\mathrm{R}$ & - & $-1070.00 \pm 0.66$ & $-1064.22 \pm 0.49$ & ASCC- $2.5 \mathrm{~V} 3$ \\
\hline 61 Vir B & - & 365.42 & $-31.65 \pm 3.71$ & $-13.56 \pm 2.26$ & ASCC-2.5 V3 \\
\hline 70 Vir A & $\mathrm{R}$ & - & $-234.8 \pm 0.7$ & $-576.1 \pm 0.5$ & Nomad-1 \\
\hline 70 Vir B & - & 268.29 & $3.9 \pm 1.3$ & $8.7 \pm 0.9$ & Nomad-1 \\
\hline$\epsilon$ Tau A & $\mathrm{R}$ & - & $107.2 \pm 1.0$ & $-36.7 \pm 0.8$ & Nomad-1 \\
\hline$\epsilon$ Tau B & - & 189.16 & $23.1 \pm 1.5$ & $-18.9 \pm 1.9$ & Nomad-1 \\
\hline$\kappa \mathrm{CrB} \mathrm{A}$ & & - & $-8.0 \pm 0.5$ & $-347.4 \pm 0.6$ & Nomad-1 \\
\hline$\kappa \mathrm{CrB} \mathrm{B}$ & & 112.31 & $-3.8 \pm 5.4$ & $-20.4 \pm 5.4$ & Nomad-1 \\
\hline$\tau$ Gem A & $\mathrm{C}$ & - & $-31.0 \pm 1.0$ & $-48.3 \pm 0.5$ & Nomad-1 \\
\hline$\tau$ Gem B & - & & \multicolumn{3}{|c|}{ proper motion not known } \\
\hline$\tau \mathrm{Gem} \mathrm{C}$ & - & 59.91 & $-64.1 \pm 9.0$ & $-12.5 \pm 9.0$ & Nomad-1 \\
\hline HIP 75458 A & $\mathrm{R}$ & - & $-8.2 \pm 0.3$ & $17.3 \pm 0.4$ & Nomad-1 \\
\hline HIP 75458 B & - & 253.77 & $0.5 \pm 1.1$ & $-5.5 \pm 1.3$ & Nomad-1 \\
\hline HD $33564 \mathrm{~A}$ & $\mathrm{R}$ & - & $-79.22 \pm 0.52$ & $161.22 \pm 0.62$ & ASCC- $-2.5 \mathrm{~V} 3$ \\
\hline HD 33564 B & - & 24.47 & $52.00 \pm 1.85$ & $-156.6 \pm 2.99$ & ASCC-2.5 V3 \\
\hline HD $62509 \mathrm{~A}$ & $\mathrm{R}$ & - & $-625.6 \pm 1.0$ & $-45.9 \pm 0.5$ & Nomad-1 \\
\hline HD $62509 \mathrm{~B}$ & - & 30 & \multicolumn{3}{|c|}{ proper motion not known } \\
\hline HD $62509 \mathrm{CD}$ & - & 248.98 & $-4.5 \pm 1.0$ & $-3.7 \pm 1.3$ & Nomad-1 \\
\hline HD $62509 \mathrm{E}$ & - & 281.26 & $-3.1 \pm 0.7$ & $-14.0 \pm 0.8$ & Nomad-1 \\
\hline HD $62509 \mathrm{~F}$ & - & 304.84 & $-6.5 \pm 0.7$ & $-2.3 \pm 0.8$ & Nomad-1 \\
\hline HD $62509 \mathrm{G}$ & - & 152.77 & $6.0 \pm 2.0$ & $-4.5 \pm 1.3$ & Nomad-1 \\
\hline HD $81688 \mathrm{~A}$ & $\mathrm{R}$ & - & $-6.4 \pm 0.6$ & $-128.1 \pm 0.5$ & Nomad-1 \\
\hline HD 81688 B & - & 71.79 & $-15.0 \pm 1.0$ & $-43.8 \pm 1.1$ & Nomad-1 \\
\hline HD $81688 \mathrm{C}$ & - & 84.05 & $-17.6 \pm 1.1$ & $-42.6 \pm 0.7$ & Nomad-1 \\
\hline HD $102365 \mathrm{~A}$ & $\mathrm{R}$ & - & $-1530.55 \pm 0.67$ & $402.73 \pm 0.62$ & ASCC- $2.5 \mathrm{~V} 3$ \\
\hline HD 102365 B & - & 23.95 & $51.6 \pm 4.4$ & $-5.1 \pm 4.3$ & UCAC-3 \\
\hline HD 110014 A & $\mathrm{R}$ & - & $-76.90 \pm 0.54$ & $-24.01 \pm 0.55$ & ASCC- $2.5 \mathrm{~V} 3$ \\
\hline HD 110014 B & - & 176.06 & $-27.19 \pm 1.82$ & $-18.01 \pm 1.80$ & ASCC- $2.5 \mathrm{~V} 3$ \\
\hline HD $110014 \mathrm{C}$ & - & 227.06 & $-9.09 \pm 1.44$ & $-1.29 \pm 2.75$ & ASCC-2.5 V3 \\
\hline HD $110014 \mathrm{D}$ & - & 320.44 & $-26.04 \pm 1.26$ & $-0.94 \pm 0.64$ & ASCC-2.5 V3 \\
\hline HD $121504 \mathrm{~A}$ & $\mathrm{R}$ & - & $-250.5 \pm 0.7$ & $-84.0 \pm 0.8$ & Nomad-1 \\
\hline HD 121504 B & - & 36.32 & $-15.0 \pm 1.5$ & $2.3 \pm 1.4$ & Nomad-1 \\
\hline HD $164922 \mathrm{~A}$ & $\mathrm{R}$ & - & $389.7 \pm 0.5$ & $-602.4 \pm 0.5$ & Nomad-1 \\
\hline HD 164922 B & - & 96.39 & $6.4 \pm 5.1$ & $-3.9 \pm 5.1$ & Nomad-1 \\
\hline HD $164922 \mathrm{C}$ & - & 93.09 & $-40.9 \pm 5.2$ & $-56.2 \pm 4.1$ & UCAC-3 \\
\hline HD 192263 A & $\mathrm{R}$ & - & $-63.3 \pm 1.6$ & $262.2 \pm 0.7$ & Nomad-1 \\
\hline HD 192263 BC & - & 72.38 & $13.6 \pm 1.2$ & $0.6 \pm 1.7$ & Nomad-1 \\
\hline HD 192263 D & - & 78.44 & $-4.3 \pm 5.6$ & $-7.9 \pm 5.6$ & Nomad-1 \\
\hline
\end{tabular}


T. Roell et al.: Extrasolar planets in stellar multiple systems $(R N)$

Table A.3. Exoplanet host stars with companion candidates, but further epoch observations are needed (same columns as in A.1).

\begin{tabular}{|c|c|c|c|c|c|}
\hline System & EPE planet status & $r\left[{ }^{\prime \prime}\right]$ & 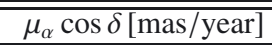 & 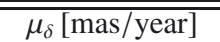 & Catalogue \\
\hline WASP-2 A & $\mathrm{S}(\mathrm{R})$ & & $4.9 \pm 3.9$ & $-50.9 \pm 6.7$ & UCAC-3 \\
\hline WASP-2 B & - & \multicolumn{4}{|c|}{ B is $0.76^{\prime \prime}$ away from A, second epoch needed, see Daemgen et al. (2009) } \\
\hline TrES & $S(R)$ & \multirow{2}{*}{\multicolumn{4}{|c|}{ 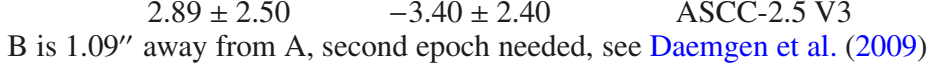 }} \\
\hline TrES-2 B & - & & & & \\
\hline TrES-4 A & $\mathrm{S}(\mathrm{R})$ & & $-8.09 \pm 4.80$ & $-33.00 \pm 4.40$ & ASCC-2.5 V3 \\
\hline TrES-4 B & - & \multicolumn{4}{|c|}{ B is $1.56^{\prime \prime}$ away from A, second epoch needed, see Daemgen et al. (2009) } \\
\hline
\end{tabular}

Table A.4. Extrasolar planets detected with transit or RV observations in closer binaries with a projected stellar separation of $\rho_{\text {app }}^{\star} \leq 1000 \mathrm{AU}$, sorted by an increasing stellar separation. For the four closest systems a value for the binary semi-major axis $\left(a_{\text {bin }}\right)$ is known from multi-epoch observations (listed in brackets in the $\rho_{\text {app }}^{\star}$ column). If RV and transit measurements are available the true mass of the exoplanet candidate is given in the table.

a... New system compared to the latest published overview by Mugrauer \& Neuhäuser (2009).

C... B component is a brown dwarf, see Mugrauer et al. (2006b).

※ ... B component is a white dwarf, see Mugrauer \& Neuhäuser (2005).

$\S$... B component is a white dwarf, see Chauvin et al. (2007).

$\dagger \ldots$ Reffert \& Quirrenbach (2011) determined an astrometric mass range for the planet candidate, which is given within the brackets in the planetary mass column.

\begin{tabular}{|c|c|c|c|c|c|c|}
\hline Note & Host star & 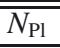 & 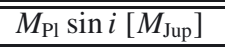 & $\overline{a_{\mathrm{Pl}}[\mathrm{AU}]}$ & $\rho_{\text {app }}^{\star}[\mathrm{AU}]$ & Reference \\
\hline+ & $\gamma$ Cephei A & 1 & $1.6(5 \ldots 27)$ & 2.04 & $12.4\left(a_{\mathrm{bin}}=20.2\right)$ & $\begin{array}{c}\text { Campbell et al. (1988); Hatzes et al. (2003) } \\
\text { Neuhäuser et al. (2007); Reffert \& Quirrenbach (2011) }\end{array}$ \\
\hline \multirow[t]{10}{*}{ ※ } & G1 $86 \mathrm{~A}$ & 1 & 4.01 & 0.11 & $20.7\left(a_{\text {bin }}=21\right)$ & Queloz et al. (2000); Mugrauer \& Neuhäuser (2005) \\
\hline & HD 41004 A & 1 & 2.54 & 1.64 & $21.5\left(a_{\text {bin }}=20\right)$ & Zucker et al. (2004); Raghavan et al. (2006) \\
\hline & HD 41004 B & 1 & 18.40 & 0.02 & $21.5\left(a_{\mathrm{bin}}=20\right)$ & Zucker et al. (2003); Raghavan et al. (2006) \\
\hline & HD 196885 A & 1 & 2.58 & 2.37 & $23.1\left(a_{\mathrm{bin}}=21\right)$ & $\begin{array}{c}\text { Correia et al. (2008); Fischer et al. (2009) } \\
\text { Chauvin et al. (2011) }\end{array}$ \\
\hline & $\tau$ Boo A & 1 & 3.90 & 0.05 & 45.2 & Butler et al. (1997); Raghavan et al. (2006) \\
\hline & GJ $3021 \mathrm{~A}$ & 1 & 3.37 & 0.49 & 68.6 & Naef et al. (2001b); Mugrauer et al. (2007a) \\
\hline & HD $177830 \mathrm{~A}$ & 1 & 1.28 & 1.00 & 100.3 & Vogt et al. (2000); Eggenberger et al. (2007) \\
\hline & HD $142 \mathrm{~A}$ & 1 & 1.03 & 1.00 & 105.0 & Tinney et al. (2002); Raghavan et al. (2006) \\
\hline & HD 114762 A & 1 & 11.02 & 0.30 & 134.0 & Latham et al. (1989); Mugrauer et al. (2005) \\
\hline & HD 195019 A & 1 & 3.70 & 0.14 & 149.2 & Fischer et al. (1999); Raghavan et al. (2006) \\
\hline \multirow[t]{3}{*}{ a } & $\gamma^{1}$ Leo A & 1 & 8.78 & 1.19 & 165.6 & Han et al. (2010) \\
\hline & HD 189733 A & 1 & 1.13 (true mass) & 0.03 & 220.0 & Bouchy et al. (2005); Eggenberger et al. (2007) \\
\hline & HD $16141 \mathrm{~A}$ & 1 & 0.23 & 0.35 & 222.6 & Marcy et al. (2000); Mugrauer et al. (2005) \\
\hline \multirow[t]{2}{*}{ a } & HD 185269 A & 1 & 0.94 & 0.08 & 226.9 & Johnson et al. (2006); Ginski et al. (2012) \\
\hline & HD $212301 \mathrm{~A}$ & 1 & 0.45 & 0.04 & 233.2 & Lo Curto et al. (2006); Mugrauer \& Neuhäuser (2009) \\
\hline$\S$ & HD $27442 \mathrm{~A}$ & 1 & 1.28 & 1.18 & 238.4 & $\begin{array}{l}\text { Butler et al. (2001); Chauvin et al. (2006) } \\
\text { Raghavan et al. (2006); Mugrauer et al. (2007a) }\end{array}$ \\
\hline \multirow[t]{3}{*}{ a } & HD $28254 \mathrm{~A}$ & 1 & 1.16 & 2.25 & 241.7 & Naef et al. (2010) \\
\hline & HD 114729 A & 1 & 0.82 & 2.08 & 283.5 & Butler et al. (2003); Mugrauer et al. (2005) \\
\hline & HD 46375 A & 1 & 0.25 & 0.04 & 345.7 & Marcy et al. (2000); Mugrauer et al. (2006a) \\
\hline$a$ & WASP-8 A & 1 & 2.25 (true mass) & 0.08 & 348.0 & Queloz et al. (2010) \\
\hline \multirow[t]{6}{*}{$a, \mathbb{C}$} & HD $3651 \mathrm{~A}$ & 1 & 0.20 & 0.28 & 478.4 & Fischer et al. (2003a); Mugrauer et al. (2006b) \\
\hline & HD 109749 A & 1 & 0.28 & 0.06 & 495.6 & Fischer et al. (2006); Desidera \& Barbieri (2007) \\
\hline & $\begin{aligned} & \text { HD } 99492 \\
= & \text { HD } 99491 \mathrm{~B}\end{aligned}$ & 1 & 0.11 & 0.12 & 589.4 & Marcy et al. (2005b); Raghavan et al. (2006) \\
\hline & HD 75289 A & 1 & 0.42 & 0.05 & 621.4 & Udry et al. (2000); Mugrauer et al. (2004a) \\
\hline & HD 188015 A & 1 & 1.26 & 1.19 & 676.0 & Marcy et al. (2005b); Raghavan et al. (2006) \\
\hline & $v$ And $\mathrm{A}$ & 3 & $0.69 \ldots 11.6$ & $0.06 \ldots 2.55$ & 742.5 & Butler et al. (1999); Lowrance et al. (2002) \\
\hline$a$ & GJ 676 A & 1 & 4.9 & 1.82 & 788.9 & Forveille et al. (2011) \\
\hline \multirow[t]{2}{*}{$a$} & HD 137388 A & 1 & 0.22 & 0.89 & 809.4 & Dumusque et al. (2011) \\
\hline & HD $142022 \mathrm{~A}$ & 1 & 4.40 & 2.80 & 890.8 & Eggenberger et al. (2006); Raghavan et al. (2006) \\
\hline$a$ & $11 \mathrm{Com} \mathrm{A}$ & 1 & 19.4 & 1.29 & 999.0 & Liu et al. (2008) \\
\hline
\end{tabular}


Table A.5. Extrasolar planets detected with transit or RV observations in wider binaries with a projected stellar separation of $\left.\rho_{\mathrm{app}}^{\star}>1000 \mathrm{AU}\right)$, sorted by an increasing stellar separation. If RV and transit measurements are available the true mass of the exoplanet candidate is given in the table.

a... New system compared to the latest published overview by Mugrauer \& Neuhäuser (2009).

I ... Closer component listed in the CCDM (formerly called B) was disproved by proper motion measurements, but a new wide stellar companion was found and confirmed by common proper motion (Mugrauer et al. 2005).

$\S$... B component is a white dwarf, see Porto de Mello \& da Silva (1997).

\begin{tabular}{|c|c|c|c|c|c|c|}
\hline Note & Host star & $N_{\mathrm{Pl}}$ & $M_{\mathrm{Pl}} \sin i\left[M_{\mathrm{Jup}}\right]$ & $a_{\mathrm{Pl}}[\mathrm{AU}]$ & $\rho_{\mathrm{app}}^{\star}[\mathrm{AU}]$ & Reference \\
\hline & HD 11964 A & 2 & 0.11 and 0.61 & 0.23 and 3.34 & 1044 & Butler et al. (2006); Raghavan et al. (2006) \\
\hline & $55 \mathrm{Cnc} \mathrm{A}$ & 5 & $0.02 \ldots 3.84$ & $0.04 \ldots 5.77$ & 1053 & Butler et al. (1997); Marcy et al. (2002); McArthur et al. (2004) \\
\hline & HD $80606 \mathrm{~A}$ & 1 & 3.94 (true mass) & 0.45 & 1197 & $\begin{array}{l}\text { Naef et al. (2001a); Moutou et al. (2009) } \\
\text { Raghavan et al. (2006); Pont et al. (2009) }\end{array}$ \\
\hline \multirow[t]{3}{*}{$a$} & HD $204941 \mathrm{~A}$ & 1 & 0.27 & 2.56 & 1447 & Dumusque et al. (2011) \\
\hline & HAT-P-1 B & 1 & 0.52 (true mass) & 0.06 & 1557 & Bakos et al. (2007) \\
\hline & HD $101930 \mathrm{~A}$ & 1 & 0.30 & 0.30 & 2227 & Lovis et al. (2005); Mugrauer et al. (2007b) \\
\hline \multirow[t]{3}{*}{$a$} & HD 7449 A & 2 & 1.11 and 2.00 & 2.30 and 4.96 & 2348 & Dumusque et al. (2011) \\
\hline & HD 89744 A & 1 & 7.99 & 0.89 & 2457 & Korzennik et al. (2000); Mugrauer et al. (2004b) \\
\hline & HD $190360 \mathrm{~A}$ & 2 & 0.06 and 1.50 & 0.13 and 3.92 & 3293 & Naef et al. (2003); Vogt et al. (2005); Raghavan et al. (2006) \\
\hline q & HD $213240 \mathrm{~A}$ & 1 & 4.50 & 2.03 & 3905 & Santos et al. (2001); Mugrauer et al. (2005) \\
\hline$\S$ & HD $147513 \mathrm{~A}$ & 1 & 1.00 & 1.26 & 4460 & $\begin{array}{c}\text { Mayor et al. (2004); Mugrauer \& Neuhäuser (2005) } \\
\text { Porto de Mello \& da Silva (1997) }\end{array}$ \\
\hline \multirow{5}{*}{$a$} & HD $222582 \mathrm{~A}$ & 1 & 7.75 & 1.35 & 4595 & Vogt et al. (2000); Raghavan et al. (2006) \\
\hline & $\mathrm{XO}-2 \mathrm{~A}$ & 1 & 0.57 (true mass) & 0.04 & 4619 & Burke et al. (2007) \\
\hline & HD $125612 \mathrm{~A}$ & 3 & $0.06 \ldots 7.2$ & $0.05 \ldots 4.2$ & 4752 & $\begin{array}{l}\text { Fischer et al. (2007); Lo Curto et al. (2010) } \\
\text { Mugrauer \& Neuhäuser (2009) }\end{array}$ \\
\hline & HD $20782 \mathrm{~A}$ & 1 & 1.90 & 1.38 & 9133 & Jones et al. (2006); Desidera \& Barbieri (2007) \\
\hline & HD 38529 A & 2 & 0.78 and 17.70 & 0.13 and 3.69 & 11915 & Fischer et al. (2001, 2003b); Raghavan et al. (2006) \\
\hline
\end{tabular}

Table A.6. Extrasolar planets detected with transit or RV observations in stellar systems with more than two components, sorted by the increasing projected separation of the host star and the nearest stellar component $\left(\rho_{\text {app }}^{\star}\right)$. For the closest systems a value for the binary semi-major axis $\left(a_{\text {bin }}\right)$ is known from multi-epoch observations (listed in brackets in the $\rho_{\text {app }}^{\star}$ column). If RV and transit measurements are available the true mass of the exoplanet candidate is given in the table.

a ... New system compared to the latest published overview by Mugrauer \& Neuhäuser (2009).

\begin{tabular}{|c|c|c|c|c|c|c|c|}
\hline Note & Host-star & $N_{\mathrm{PI}}$ & $N_{\star}$ & $M_{\mathrm{Pl}} \sin i\left[M_{\mathrm{Jup}}\right]$ & $a_{\mathrm{PI}}[\mathrm{AU}]$ & $\rho_{\mathrm{app}}^{\star}[\mathrm{AU}]$ & Reference \\
\hline \multirow[t]{2}{*}{$\bar{a}$} & HD $126614 \mathrm{~A}$ & 1 & 3 & 0.38 & 2.35 & 36.2 & Howard et al. (2010); Ginski et al. (2012) \\
\hline & HD 19994 A & 1 & 3 & 1.68 & 1.42 & 51.5 & $\begin{array}{c}\text { Mayor et al. (2004); Raghavan et al. (2006) } \\
\text { Roell et al. (2011) }\end{array}$ \\
\hline \multirow[t]{2}{*}{ a } & GJ $667 \mathrm{C}$ & 2 & 3 & 0.018 and 0.014 & 0.05 and 0.12 & 227.0 & Anglada-Escudé et al. (2012) \\
\hline & HD 65216 A & 1 & 3 & 1.21 & 1.37 & 256.3 & Mayor et al. (2004); Mugrauer et al. (2007b) \\
\hline \multirow[t]{4}{*}{ a } & HD 132563 B & 1 & 3 & 1.49 & 2.62 & 365.2 & Desidera et al. (2011) \\
\hline & HD $196050 \mathrm{~A}$ & 1 & 3 & 3.00 & 2.50 & 511.2 & $\begin{array}{c}\text { Jones et al. (2002); Mugrauer et al. (2005) } \\
\text { Eggenberger et al. (2007) }\end{array}$ \\
\hline & HD $178911 \mathrm{~B}$ & 1 & 3 & 6.29 & 0.32 & 794.3 & Zucker et al. (2002); Eggenberger et al. (2003) \\
\hline & 16 Cyg B & 1 & 3 & 1.68 & 1.68 & 859.7 & Cochran et al. (1997); Raghavan et al. (2006) \\
\hline \multirow[t]{2}{*}{$a$} & 30 Ari B & 1 & 3 & 9.88 & 0.995 & 1517 & Guenther et al. (2009) \\
\hline & HD 40979 A & 1 & 3 & 3.32 & 0.81 & 6395 & $\begin{array}{c}\text { Fischer et al. (2003b); Eggenberger et al. (2003) } \\
\text { Mugrauer et al. (2007a) }\end{array}$ \\
\hline
\end{tabular}

